\title{
Wital: Contactless Real-time Vital Signs Monitoring System Using COTS WiFi Devices
}

\author{
Yu Gu, Senior Member, IEEE, Xiang Zhang, Huan Yan, Zhi Liu, Senior Member, IEEE, \\ Fuji Ren, Senior Member, IEEE
}

\begin{abstract}
Real-time vital signs (breathing and heartbeat) monitoring is essential for patient care and sleep disease prevention. Current solutions are mostly based on wearable sensors or cameras, the former affects the quality of sleep, while the latter is not conducive to privacy protection, and the cost of these methods is usually expensive. In this paper, we propose Wital, a real-time vital signs monitoring system based on the lowcost and widespread COTS WiFi device. Most of the existing WiFi-based vital signs monitoring solutions utilize the line of sight (LOS) signals to achieve good performance. However, in real-life environments, NLOS sensing is more common. In this article, we first model the relationship between the energy ratio of LOS/NLOS signals and the ability to monitor vital signs based on the Ricean- $K$ theory and theoretically prove that blocking LOS signals in NLOS sensing is more beneficial. Wital also proposed a motion segmentation algorithm based on motion regularity detection, which can accurately distinguish breathing from other motions. We have also established a realtime vital signs monitoring system to verify our method, and the experimental results prove the effectiveness of our method.
\end{abstract}

Index Terms-Wi-Fi, CSI, sleep monitoring, vital signs, wireless sensing

\section{INTRODUCTION}

Real-time vital signs (breathing and heartbeat) monitoring is essential for patient care and sleep disease prevention. For example, Covid-19 can cause breathing difficulties or shortness of breath in patients [1], so patients need continuous breathing monitoring. Some people may suffer from various diseases during their daily sleep, such as sleep apnea [2] and asthma [3]. Some studies have also shown that breathing disorders are the main cause of Sudden Infant Death Syndrome (SIDS) in sleeping infants [4]. In many examples of sleep disorders, patients only develop symptoms for a short period of time or occasionally, and long-term continuous monitoring is required to detect such symptoms. However, under the condition of limited medical resources caused by Covid-19 and limited funds, long-term hospital observation is unrealistic for most people. Therefore, continuous, real-time and cost-effective vital signs monitoring in the home environment is essential.

Traditional vital signs monitoring schemes mainly leverage attached sensors, such as Polysomnography (PSG) [5] and Electrocardiogram (ECG) [6]. Nevertheless, these special

Yu Gu, Xiang Zhang and Huan Yan are with the School of Computer and Information, Hefei University of Technology, China. E-mail: yugu.bruce@ieee.org, zhangxiang@mail.hfut.edu.cn

Zhi Liu was with the Dept. of Mathematical and Systems Engineering, Shizuoka University, Japan. E-mail: liu@ shizuoka.ac.jp

Fuji Ren was with the Dept. of Information Science and Intelligent Systems, Univ. of Tokushima, Japan. E-mail: ren@is.tokushima-u.ac.jp devices are not suitable for the home environment, they are expensive and impair the quality of sleep. The solutions based on pressure or acceleration sensors also require contact with the body, and lighting conditions limit solutions based on computer vision. Recently, Radio Frequency (RF) based methods [7], [8] has attracted considerable attention as they provided non-invasive vital signs monitoring use SDR (Software Defined Radio). However, the devices used in these solutions are generally expensive and have disadvantages such as infringement of privacy and discomfort to the wearer.

Recently, WiFi-based vital signs monitoring has received widespread attention [9]-[11] due to the widespread and low cost of WiFi devices. The reason why WiFi can detect vital signs is that breathing and heartbeat can cause deformations in the abdomen and chest, and these deformations can affect the propagation of WiFi signals, which can be recorded by the WiFi Channel State Information (CSI) [12].

A major challenge for Wi-Fi-based vital signs perception is that the torso deformation caused by breathing/heartbeat is extremely weak, and it is difficult to greatly affect the propagation of Wi-Fi signals. Therefore, a model is needed to guide the system implementation. Currently, state-of-theart schemes mostly based on the Fresnel Zone model [9], [13] or CSI-ratio model [10], [14] to guide the WiFi-based vital signs monitoring. For the former, the Fresnel Zone model only considered the signal reflect path (dynamic path), this model points out that the best performance can be achieved in the case of LOS or close to LOS, however, it is almost impossible to achieve in a real-life environment. For the latter, at least two antennas placed close together are required at the receiver, this is unfavorable for the single-antenna receiver and increases the calculation loss. Another challenge is that motions such as turning over can be mixed with breathing/heartbeat and affect the accuracy of vital signs detection. Therefore, breathing/heartbeat must be distinguished from other actions. However, since breathing/heartbeat and turning over are dynamic, the current dynamic and static segmentation schemes [15] used in WiFi-based perception are not applicable.

In this paper, we propose Wital, a real-time vital signs monitoring system based on low-cost and widespread COTS WiFi devices. To address the aforementioned challenges, we first propose a Ricean-K theory-based model to analyze the relationship between the energy ratio of LOS/NLOS signals and the ability to monitor vital signs, and based on the model analysis, we block the LOS signal to improve the NLOS sensing ability. We also propose a regularity-based motion segmentation algorithm to accurately separate breathing/heartbeat 
and other motions.

The main contributions of this paper are summarized as follows:

1) We propose a NLOS sensing model to analyze the relationship between the energy ratio of LOS/NLOS signals and the ability to monitor vital signs. We theoretically prove that blocking LOS signals in NLOS sensing is more conducive to motion detection. We verified this idea through experiments. Please note that this model can also be applied to other WiFi-based motion detection and recognition research.

2) We propose a motion segmentation algorithm based on regularity detection, which can accurately locate the position of motions (such as turn over and get up) different from vital signs.

3) We implement a real-time system using Matlab to evaluate our method. The experimental results indicated the performance of our method, and the accuracy is $96.618 \%$ and $94.708 \%$ for breathing and heart rate detection, respectively.

We organize the remainder of this paper as follows: in section II, we provide an overview of the related work. We describe our NLOS sensing model in section III. And we introduce our system design in section IV. Then, we evaluate our method and show the experimental results in section $\mathrm{V}$. Finally, we conclude our work in section VI.

\section{RELATED WORK}

\section{A. Sensing With WiFi}

Due to the widespread deployment of WiFi devices and the convenience of wireless sensing, research on passive sensing based on WiFi has received widespread attention [19]-[21]. These researches mainly use the Received Signal Strength Index (RSSI) or CSI. RSSI is easy to acquire, but the perceiving granularity is coarse. CSI can be obtained by modifying the underlying driver of the WiFi network card, and the perceiving granularity is better than RSSI.

With the help of WiFi RSSI or CSI information, current research can implement person detection [22], gesture recognition [23], position [24], [25], sleep movement detection [26] and driving activity detection [27], etc. Based on the passive sensing characteristics of $\mathrm{WiFi}$ signals, some tasks can be better carried out. In the past two years, WiFi-based perception researches have been further expanded into new fields. [28] use WiFi devices to image key points of the human body, enabling human visualization without vision equipment. [29] use WiFi devices to track finger draws in the air. [30] achieved steal mobile phone passwords using COTS WiFi devices.

\section{B. Breathing And Heartbeat Monitoring}

Respiratory frequency and heart rate are critical physiological indicators of the human body, and they are important indicators for evaluating health conditions. In general, the methods used to track such information can be categorized into three groups: sensors-based, vision-based, and RF signalbased.
Most of the traditional solutions use sensors for physiological signal detection, for example, Polysomnography (PSG) [5] and Electrocardiogram (ECG) [6] measures body functions like breathing or heartbeat by attaching multiple sensors to a patient. H.Aly et al. [31] utilize the accelerometer and gyroscope on the mobile phone to detect the chest breathing action of the person. Smart sleeping mat [32] uses pressure sensor arrays for breathing detection. However, sensors-based methods are usually expensive, complex to deploy, and invasive.

Vision-based solutions [33] are usually subject to light conditions and also raise privacy concerns. Recently, research on 'taking wireless radio signals as sensors' has received more and more attention. When RF signals travel from a transmitter to a receiver, it can be influenced by the breathing-induced chest movement on the propagation paths. RF signal-based solutions are usually based on special equipment, such as ultrawideband [34] and Frequency Modulated Continuous Wave (FMCW) radar [7], [8]. The devices used in these solutions are expensive and not suitable for daily environments. Compared with these solutions, WiFi-based solutions are less expensive and simple to deploy and can be implemented using standard equipment.

Previous WiFi-based breathing monitoring researches are mostly based on RSSI [35]-[37]. RSSI characterizes the total received power of all paths, which is coarse-grained and inherently incapable of capturing the multipath effect. In contrast, CSI can capture fine-grained channel information and multipath information well. Therefore, the latest related schemes are mostly based on CSI [9], [10], [12], [13], [16], [38], [39]. In particular, Liu et al. [16] obtain respiratory rate by using Short-Time Fourier Transform (STFT) on the CSI amplitude, it can obtain breathing information in different sleeping postures. Nevertheless, this solution needs to deploy two routers and three computers. The authors of [12] use a pair of devices to monitor the breathing rate in different sleeping postures. However, they need the line of sight between the WiFi device and access point crossing the person's chest.

The authors of [17] use the CSI phase to detect breathing rate, they use cables and splitters to connect the transmitter and receiver, and need to process two receiver data streams to eliminate phase errors. [9] calculates the best position to detect respiration by deriving the Fresnel Zone model. However, Fresnel Zone theory is limited by other factors such as obstacles in the real environment. [10] uses the CSI-ratio of two receive antennas to eliminate the phase offset and utilize the complex plane projection to achieve long-distance breathing detection. However, at least two antennas placed close together are required at the receiver, this is unfavorable for the single-antenna receiver and increases the calculation loss. [18] is the first to use a mobile phone to achieve respiratory monitoring, they propose an ambient reflection signal model under the NLoS setting to obtain CSI amplitude variations at the receiver which vary with the fine-grained displacement of the human chest, and shows that blocking the LOS signal is indeed beneficial to NLOS sense, but no theoretical proof is provided. We compare these systems and our work in Table I. 
TABLE I: Compare the latest research with our system.

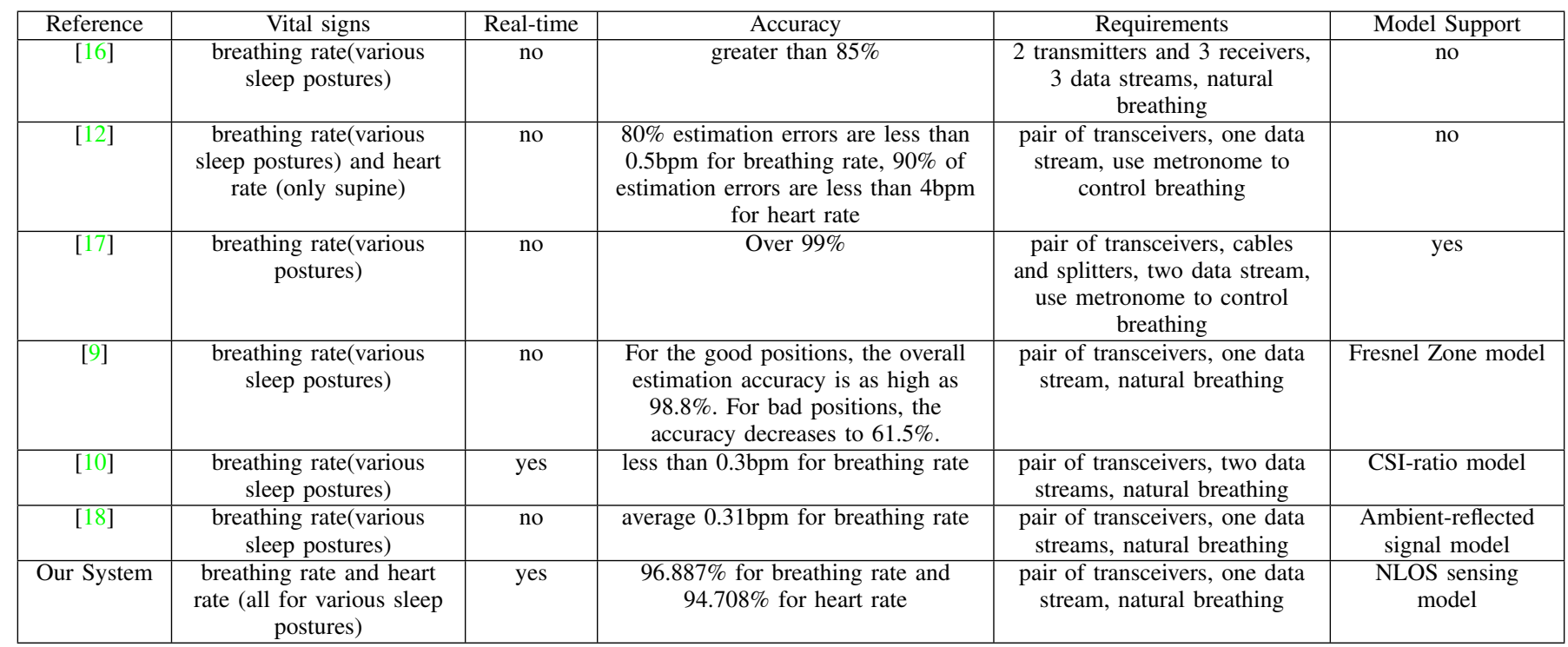

Note that this paper is an extension of our previous work [40]. In [40], we found that blocking the LOS signal is beneficial to NLOS sensing, and in this paper, we use the Ricean-K theory-based model to explain why. We also propose a new motion segmentation algorithm based on regularity detection, which can accurately locate the sleep motions (such as turn over and get up) different from breathing/heartbeat. We also improve the related work section and the experimental section over our previous work.

\section{Preliminaries}

In this part, we analyze the preliminary experimental phenomena to explain why the WiFi sensing method based on the Fresnel zone model is limited. Then, we propose our NLOS sensing model based on the Ricean-K theory.

\section{A. Channel State Information}

CSI describes the signal's attenuation on its propagation paths, such as scattering, multi-path fading or shadowing fading caused by motions, and power decay over distance. In the frequency domain, it can be characterized as:

$$
Y=H \cdot X+N
$$

Where $Y$ and $X$ are the received and transmitted signal vectors, respectively. $N$ is the additive white Gaussian noise, and $H$ is the channel matrix representing CSI information.

For WiFi CSI, the received signal's CFR (channel frequency response) can be expressed simply as the superposition of dynamic path CFR and static CFR, and it can be represented as:

$$
H(f, t)=H_{s}(f, t)+H_{d}(f, t)
$$

The dynamic CFR can be written as:

$$
H_{d}(f, t)=\sum_{k \in D} h_{k}(f, t) e^{-j 2 \pi f \tau_{k}(t)}
$$

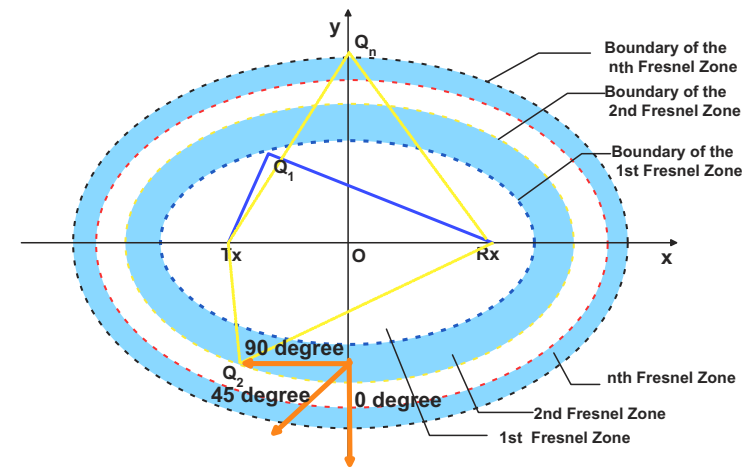

Fig. 1: Fresnel Zone

where $f$ and $\tau_{k}(t)$ represent the carrier frequency and the propagation delay on the $k^{\text {th }}$ path, respectively. $D$ is the set of dynamic paths. The received signal has a time-varying amplitude in complex plane [13]:

$$
|H(f, \theta)|^{2}=\left|H_{s}(f)\right|^{2}+\left|H_{d}(f)\right|^{2}+2\left|H_{s}(f)\right|\left|H_{d}(f)\right| \cos \theta
$$

$\theta$ is the phase difference between the static vector and the dynamic vector, the part that causes the amplitude fluctuation of the CSI waveform is $2\left|H_{s}(f)\right|\left|H_{d}(f)\right| \cos \theta$. In the case where the range and position of the motion are constant, $\theta$ is constant, and the factor affecting the fluctuation range is $\left|H_{s}(f)\right|$ and $\left|H_{d}(f)\right|$.

\section{B. Effectiveness And Limitation Of The Fresnel Model Based WiFi Sensing}

The CSI-ratio model requires at least two receiving antennas close together, the ambient-reflected signal model mainly studies the ambient-reflected signals, which are inconsistent with our system goals. Therefore, we chose the Fresnel Zone model as a guide for the preliminary experiments. As shown in Fig. 1, Fresnel zones are defined as a series of concentric ellipsoids, and $P_{1}$ and $P_{2}$ are the positions of the transmitting antenna 


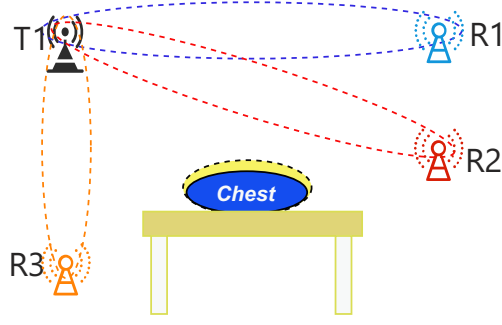

(a)

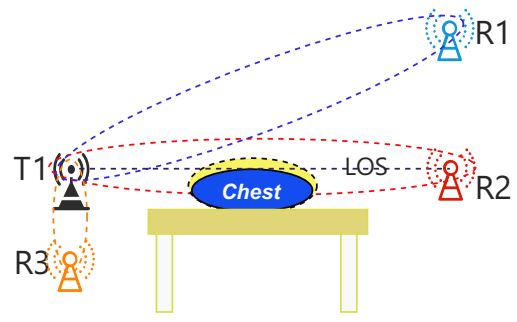

(b)

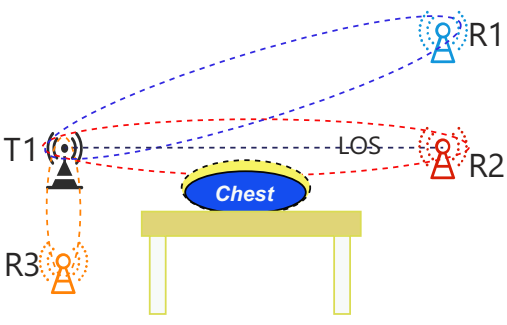

(c)

Fig. 2: The antenna settings for the preliminary experiments. The elliptical area between the antennas is the FFZ (First Fresnel Zone) range. The part between the solid chest line and the dotted line is the chest deformation range caused by breathing. (a)Setting1, T1 is the transmit antenna and R1, R2, R3 are the receive antennas, the distance from T1-R3 is 80cm, T1-R1 is $120 \mathrm{~cm}$; (b) Setting2, the chest is on the LOS of T1-R2, the distance from T1-R3 is 20cm, T1-R2 is 120cm; (c) Setting3, the chest is in the FFZ of T1-R2, the distance from T1-R3 is $30 \mathrm{~cm}, \mathrm{~T} 1-\mathrm{R} 2$ is $120 \mathrm{~cm}$.

and receiving antenna, respectively. $T x$ and $R x$ represent the sender and receiver, respectively. For a given radio wavelength $\lambda$, we could construct Fresnel zones by the following equation [41]:

$$
\left|T x Q_{n}\right|+\left|Q_{n} R x\right|-|T x R x|=n \lambda / 2
$$

where $Q_{n}$ is a point at the boundary of the $n$th Fresnel zone.

The Fresnel zone model only considers CSI changes caused by dynamic path changes. When the human body motion causes the effective displacement $d(t)$ (the effective displacement represents the change in the length of the signal reflection path), the dynamic path phase shift caused by the motion can be indicate as $e^{-j 2 \pi f \tau_{k}(t)}=e^{-j 2} \operatorname{pid}(T) / \lambda$, where $\lambda$ represents the wavelength of the $\mathrm{WiFi}$ signal. The greater the effective displacement of the motion, the greater the phase shift caused by the motion, and the more obvious the response to the $\left|H_{d}(f)\right|$ and the CSI.

Previous studies have shown that when the human body deformation direction is 0 degrees, the effective displacement caused by motion is the largest, and the sensing efficiency is the best. As the angle increases, the effective displacement becomes smaller, and the sensing efficiency becomes worse [13]. Moreover, the closer a human is to the LOS of Tx/Rx, the better sensing performance is [9]. Based on these findings, we construct a prototype system to carry out some preliminary experiments.

[Prototype] Our prototype system composes of two commodities MiniPCs, which are all equipped with an Intel Network Interface Controller 5300 and are the transmitting and receiving device, respectively. Antennas settings are shown in Fig. 2.

[Participant] One 22 year-old student participated in preliminaries experiments.

[Environment] We conduct the experiments in a $7 \times 10 \mathrm{~m}^{2}$ office room, with the furniture including chairs, couches, computer desks, and book cabinets, as shown in Fig. 5.

[Setting] The package sending rate is set to $1000 \mathrm{~Hz}$, participant conducted the experiment with different sleeping postures (prone, supine, facing left recumbent and facing right recum- bent) with different antenna setting (as shown in Fig. 2), the experiments results as shown in Fig. 3.

We analyze the preliminary results and obtain the following key observations [40]:

Breathing indeed affects channel response and experimental setting affects the channel response: Firstly, we confirm that breathing in terms of signal variations has been recorded by all settings. As shown in Figure. 3, we can observe obvious fluctuations in CSI amplitude caused by breathing in all settings.

Fresnel Zone model based sensing is effective: As shown in Fig. $3 \mathrm{~b}$ and 3c, it's hard to observe significant fluctuation caused by breathing with some sleeping postures sometimes. And based on the Fresnel Zone model, we can give reasonable explanations for some of these cases. For example, the T1-R2 antenna pair has poor perception when the volunteer is facing left/right recumbent. It owing to the deformation direction of the torso in anteroposterior dimension (breathing mainly cause deformation in this dimension) is almost parallel to the LOS path of T1-R2, thus, the deformation of the trunk can only cause very weak dynamic path changes (the length of dynamic path does not change when moving along the ellipse border of Fresnel Zone.). It is hard to cause obvious effective displacement according to the Fresnel zone model [13]. And the abdominal/thoracic deformation during respiration in the mediolateral dimension is too small(when facing left/right recumbent, deformation in mediolateral dimension is perpendicular to T1-R2, however, such deformation area is too small). And similar to [9], We have also observed that getting close to the LOS can indeed get excellent sensing performance (For supine and prone in setting2).

Fresnel Zone model based sensing is limited: However, the Fresnel zone model cannot explain some phenomena in our experiments. For setting 1, when a human is prone or supine, the direction of abdominal/thoracic deformation in anteroposterior dimension caused by breathing is nearly parallel to T1-R3. According to the Fresnel model, the effective displacement of chest/abdomen is tiny, and the performance of T1-R3 sensing should not be excellent, at least it should be worse than T1-R1 sensing, however, T1-R3 sensing performance better than T1- 


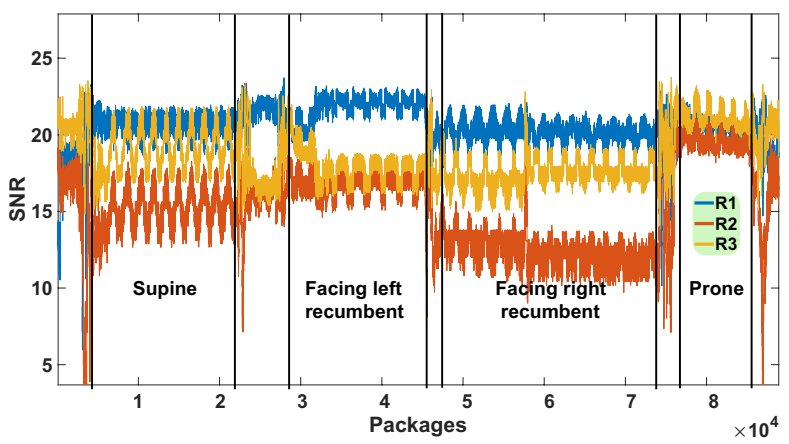

(a)

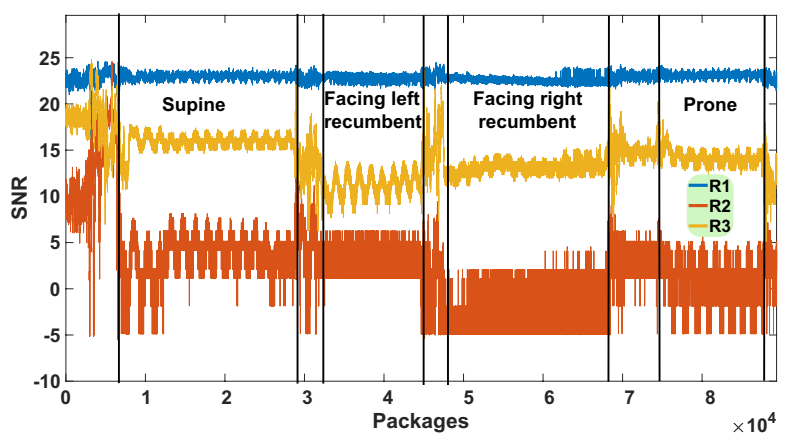

(b)

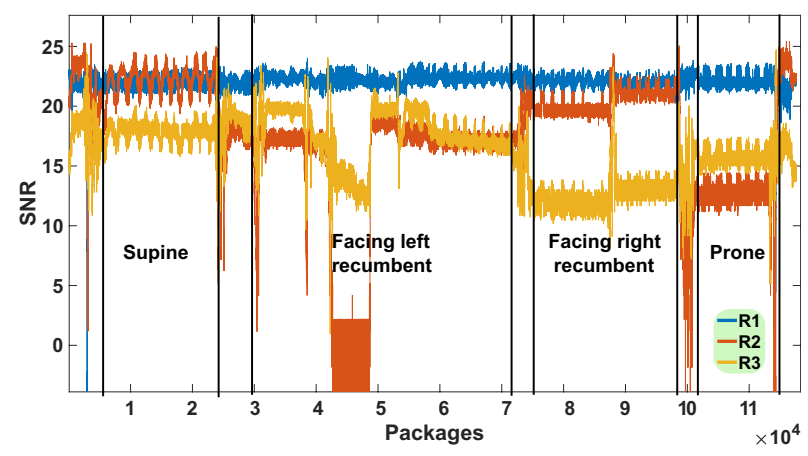

(c)

Fig. 3: (a) Detection results of setting 1; (b) Detection results of setting 2; (c) Detection results of setting 3.

$\mathrm{R} 1$. We believe that the reason for the above phenomenon may be that the LOS path of the T1-R3 antenna pair is blocked by the shelf. And we approve our guess with RiceanK-based derive and experiments. Moreover, the Fresnel Zone model believes that the sensing performance is best when the sensing object is close to the LOS path, however, in real-life deployments, NLOS sensing is more common, and there are limitations in bringing the perception object close to the LOS path.

\section{NLOS Sensing Model Based On Ricean-K}

In this section, we propose our NLOS sensing model to analyze the relationship between the power ratio of LOS/NLOS signals and the NLOS sensing ability based on the Ricean-K factor.

The Ricean $\mathrm{K}$ factor is defined as the ratio of the power in the LOS path to the power in the NLOS path. The baseband in-phase/quadrature-phase (I/Q) representation of the received signal can be expressed as follows [42]:

$$
x(t)=\sqrt{\frac{K \Omega}{K+1}} e^{j\left(2 \Pi f_{D} \cos \left(\theta_{0}\right) t\right)+\phi_{0}}+\sqrt{\frac{\Omega}{K+1}} h(t)
$$

Here $K$ is the Ricean factor, $\Omega$ denotes the total received power, $\theta_{0}$ and $\phi_{0}$ are the Angle of Arrival (AOA) and phase of the LOS, respectively, $f_{D}$ is the maximum Doppler frequency and $h(t)$ is the diffuse component given by the sum of a large number of multipath components, constituting a complex Gaussian process.

Since antenna do not move in the experiments, ie $f_{D}=0$, we simplify Equation (6) to get:

$$
x(t)=\sqrt{\frac{K \Omega}{K+1}} e^{\phi_{0}}+\sqrt{\frac{\Omega}{K+1}} h(t)
$$

In the case where the torso does not block LOS, all LOS components and part of NLOS components belong to the static path; part of NLOS components belong to the dynamic path. Combined with Equation (7) and ignoring the transmitted power, we define $\left|H_{s}\right|$ and $\left|H_{d}\right|$ as follows:

$$
\begin{gathered}
\left|H_{S}\right|=\frac{K}{K+1}+\frac{1}{K+1} \cdot \rho \\
\left|H_{d}\right|=\frac{1}{K+1} \cdot(1-\rho)
\end{gathered}
$$

$\rho$ is the proportion of static paths in the NLOS components. Combine with Equation (4) to get the following equation:

$$
\begin{aligned}
|H|^{2}=\left|H_{S}\right|^{2}+ & \left|H_{d}\right|^{2}+2\left|H_{s}\right|\left|H_{d}\right| \cos \theta \\
& =\frac{(K+\rho)^{2}}{(K+1)^{2}}+\frac{(1-\rho)^{2}}{(K+1)^{2}} \\
& +\frac{2(K+\rho)(1-\rho)}{(K+1)^{2}} \cos \theta
\end{aligned}
$$

Signal amplitude variation caused by motion can be quantified as:

$$
f(K, \rho)=2\left|H_{s}\right|\left|H_{d}\right| \cos \theta=\frac{2(K+\rho)(1-\rho)}{(K+1)^{2}} \cos \theta
$$

The value of the above formula is related to three variables, namely $\theta, K$ and $\rho$. Consider that the change in phase difference caused by breathing is relatively stable, we omit $\theta$ without considering. Then take the derivative of equation 11 of $K$ to get the following formula:

$$
f^{\prime}(K)=\frac{2(1-\rho)\left(-K^{2}-2 \rho K+1-2 \rho\right)}{(K+1)^{4}}
$$

When $K>1-2 \rho, f(K, \rho)$ decreases as $K$ increases, under normal circumstances, only a small part of the signal of the omnidirectional antenna can be reflected by the human body, which means that $\rho$ is generally bigger than 0.5 . In other words, blocking the LOS path appropriately can make CSI more sensitive to motions.

\section{How $\rho$ influence WiFi sensing capability?}

In some WiFi-based sensing research, directional antennas can be used to help WiFi sensing, the directional antenna transmits the signal directly towards the human body, make as many NLOS components as possible belong to dynamic 


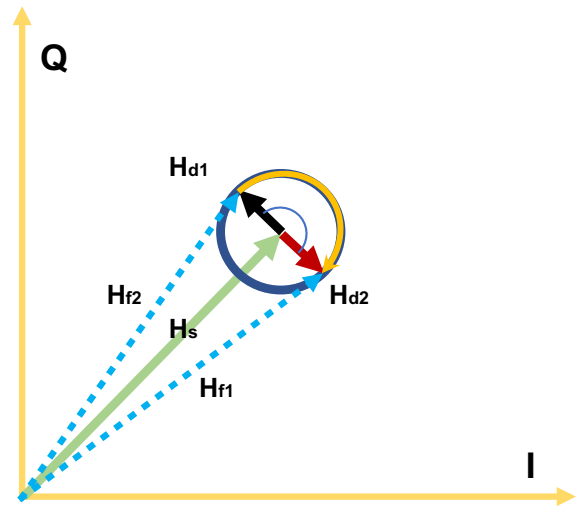

Fig. 4: When $\left|H_{s}\right|>=\left|H_{d}\right|$, CSI in the complex plane

paths (decrease $\rho$ ). We can also use equation 11 to explain why it works and provide a guide for follow-up researches.

We first use the formula to explain the influence of $\rho$ on WiFi sensing, take the derivative of equation 11 of $\rho$ to get:

$$
f^{\prime}(\rho)=\frac{2(-2 \rho+K-1)}{(K+1)^{2}}
$$

When $\mathrm{K}$ does not change, the WiFi sensing capability increases in the interval $\left[0, \frac{1-K}{2}\right]$ as $\rho$ increases, and decreases in the interval $\left[\frac{1-K}{2}, 1\right]$ as $\rho$ increases. For a more intuitive explanation, we map the CSI signal to the complex plane for discussion.

Follow [10], we first explain how to use the complex plane to intuitively "see" the motion-sensing capabilities: As shown in Figure. $4, H_{s}, H_{d}$ and $H_{f}$ represents the static, dynamic and composite of $H_{s}$ and $H_{d}$ vector, respectively. According to the research [10], when the dynamic path length is artificially changed in a short term, the amplitude of $H_{d}$ is unchanged, but the phase (The angle of $H_{d}$ relative to the $I$ axis) is changed. It means the $H_{d}$ vector is drawn a circle with the endpoint of $H_{S}$ as the center in the complex plane, as shown in Figure. 4. The amplitude and phase we extracted from CSI correspond to the amplitude of $H_{f}$ and its angle with respect to the I axis, respectively, and the capability of CSI sensing can be expressed as:

$$
A S=\left|H_{f_{\text {max }}}\right|-\left|H_{f \text { min }}\right|
$$

$A S$ is the ability of CSI sense, it is the maximum amplitude difference of the CSI waveform caused by the motion. $\left|H_{f \max }\right|$ and $\left|H_{f \min }\right|$ is the max and min absolute value of the composite vector $H_{f}$ respectively.

Next, we discuss how $\rho$ affects the sensing capability in two cases: $K>1$ and $0<=K<=1$. When $K>1$, $\frac{1-K}{2}<0$, according to equation 13 , sensing capability monotonously decreases over the interval $[0,1]$ as $\rho$ increase. In the complex plane, it indicate that $\left|H_{s}\right|>\left|H_{d}\right|$, when $\left|H_{s}\right|>=\left|H_{d}\right|,\left|H_{f \max }\right|^{2}=\left|H_{s}\right|^{2}+\left|H_{d}\right|^{2}+2\left|H_{s}\right|\left|H_{d}\right|$, and $\left|H_{f \text { min }}\right|^{2}=\left|H_{s}\right|^{2}+\left|H_{d}\right|^{2}-2\left|H_{s}\right|\left|H_{d}\right|$. The maximum value that $A S$ can reach is $\left(\mid H_{s}+H_{d}\right)-\left(\mid H_{s}-H_{d}\right)=2\left|H_{d}\right|$, in other words, the bigger $H_{d}$ is, the better sensing capability is.

When $0<=K<=1$, it means $0.5>=\frac{1-K}{2}>=0$, according to equation 13 , the sensing capability increase in the interval $\left[0, \frac{1-K}{2}\right]$ as $\rho$ increase, and decrease in the interval $\left[\frac{1-K}{2}, 1\right]$ as $\rho$ increase. In the complex plane, When $\rho$ is in the interval $\left[\frac{1-K}{2}, 1\right]$, according to equation 8 and $9,\left|H_{s}\right|>\left|H_{d}\right|$, this situation is the same as the previous paragraph, smaller $\rho$ is better. When $\rho$ is in the interval $\left[0, \frac{1-K}{2}\right]$, which is $\left|H_{s}\right|<$ $\left|H_{d}\right|$, the max value of $A S$ is $\left|H_{f \max }\right|-\left|H_{f \text { min }}\right|=\left|H_{d}\right|+$ $H_{s}\left|-\left(\left|H_{d}\right|-\left|H_{s}\right|\right)=2\right| H_{s} \mid$, in other words, the bigger $H_{s}$ is, the better sensing capability is, which means bigger $\rho$ is better.

In practical applications, it is difficult to achieve $\left|H_{S}\right|<$ $\left|H_{d}\right|$, unless $K<1$ and most of the NLOS are belong to the dynamic path. Therefore, decreasing $\rho$ is conducive to improve sensing capability. And the directional antenna can reduce $\rho$, therefore, the directional antenna is beneficial to WiFi-based sensing.

Will blocking the LOS make sensing capability worse?: Blocking the LOS path can reduce the Ricean-K value, but will blocking must improve the detection capability? Equation 11 have two main variables, $K$ and $\rho$. Whether there such a situation that block the LOS path can reduce $K$, but $\rho$ increased, results in poorer motion perception ability? We think this situation is difficult to happen, unless the occlusion makes the static signal energy $\left(\left|H_{s}\right|\right)$ reaching the receiver too small. Assuming that the worst case, which is blocking the LOS path increases the NLOS by $L$, but $L$ is not allocated to the dynamic vector at all. The amplitudes of $\left|H_{s}\right|$ and $\left|H_{d}\right|$ are unchanged, their product is unchanged, and the sensing capability equal to the original situation. In the actual indoor environment, it is difficult to make the blocked LOS signal do not propagate towards the human body at all. In summary, no matter $\rho$ is larger, smaller, or unchanged, the sensing capability will not be deteriorated by block the LOS (Unless the occlusion affects the signal received at the receiver).

\section{SYSTEM DESIGN}

\section{A. System Overview}

In this section, we introduce the system design of our realtime vital signs monitoring system, Wital. Our Wital system is shown in Fig. 5, and the system is divided into three modules:

Data Collecting. We collect better CSI data for vital signs monitoring based on our NLOS sensing model (block the LOS). Since the data collection setup is different in different scenarios, this section only describes the general data processing module (data preprocessing and vital signs extracting) in detail. And the data collection setting corresponding to this paper is explained in the evaluation section.

Data Preprocessing. We select the best performing subcarrier by subcarrier selecting first, then segment the vital signs and other motions based on our motion segmentation algorithm, finally, we denoise the CSI data to remove outliers.

Vital Signs Extracting. Data after preprocessing are divided into two parts by frequency domain segmenting, one mainly including breathing and another one mainly containing heartbeat, then we extract the breathing rate and heart rate, respectively. 


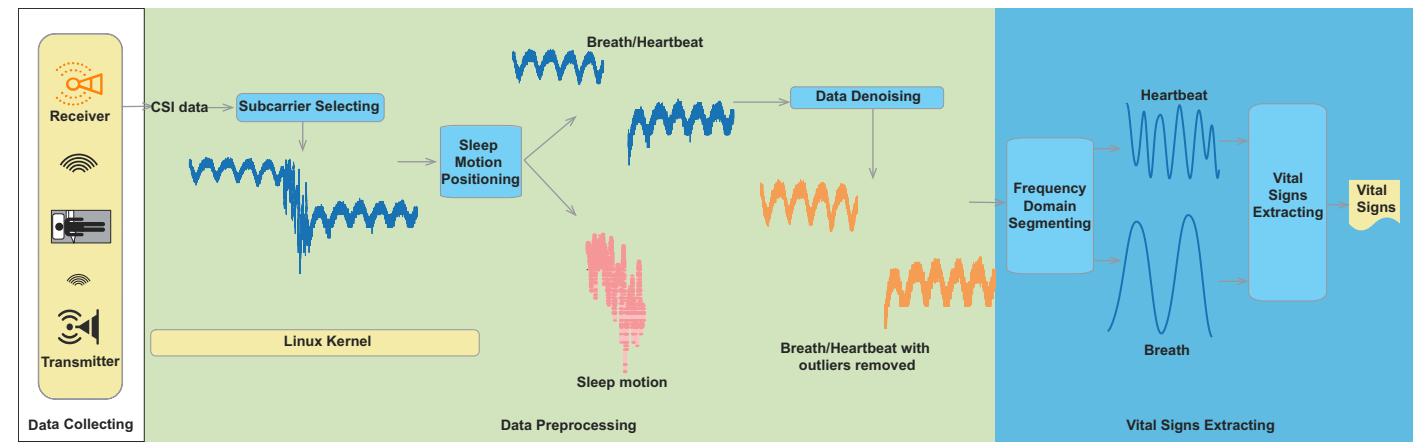

Fig. 5: System architecture

\section{B. Data Preprocessing}

Subcarrier Selection. Different subcarriers have different central frequencies and may have different sensing performances. Therefore, it is essential to choose a proper subcarrier that can better capture vital signs. According to the previous experience [40], we choose the subcarrier with the biggest variance for Wital.

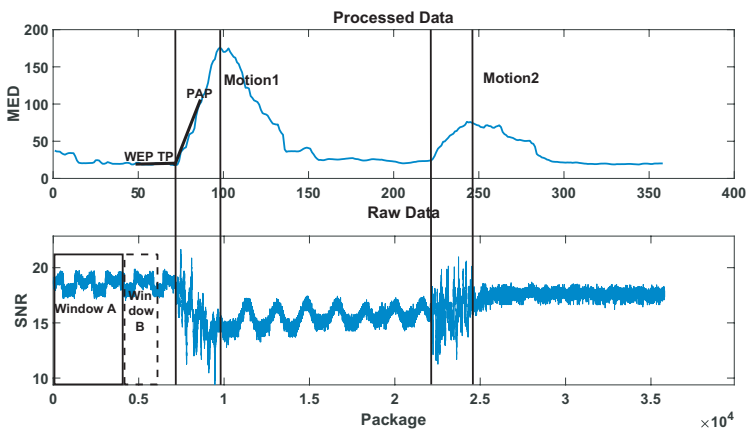

Fig. 6: Regularity calculation result

Motion Segmentation. When monitoring vital signs, the monitored subject can't remain calm all the time. Random motions such as turning over can affect the accuracy of vital signs monitoring, and it is necessary to design a motion segmentation algorithm to locate and filter these motions which different from breathing/heartbeat.

Since breathing/heartbeat and turning over are all movements, the dynamic and static segmentation method used in previous studies is not applicable. To fill in this gap, we propose a motion segmentation algorithm that can segment different types of movement, our algorithm is based on the assumption that different types of motion have different regularities. We choose an instance containing two turning over and normal breathing to verify this hypothesis, as shown in Fig. 6.

To calculate the regularity of the CSI data, we set a window $A$ with variable length and a window $B$ with fixed length first. $A$ starts at the beginning of the CSI waveform, and $B$ starts from the end of $A$, both $A$ and $B$ have an initial length of 2000 packages. Then we calculate the minimum euclidean distance (MED) from the CSI data contained in $B$ and the data in $A$ and record it. Then, make the window $A$ expand backward by 100 packets, and make the window $B$ moves backward by 100 packages, calculate the MED. Iterate like this until the end of the calculation.

The result is shown in Fig.6, when the first turning over occurs, the MED increases sharply, and when the motion ends, the MED begins to decrease. Since $A$ already contains a turning over motion, when the second turning over occurs, its MED changes are not dramatic. From the experiment result, we can observe that the regularity between different types of motion is different, and the regularity between the same types of action is similar.

The key steps of our segmentation algorithm are as follows:

1) Initialization. Set a variable-length window $A$ and a fixed-length window $B$. $A$ starts from the beginning of CSI, and $B$ starts from the end of $A$. The initial lengths of $A$ and $B$ are both 2000 packets, set up an empty collection $M A$;

2) Activation point detection. Calculate the MED of CSI as described above, if $M E D>v \cdot \operatorname{ave}(M A)$ in one iteration(ave() is averaging function), mark this point as a positioning activation point $(P A P)$, turn to the next step, else record MED into $M A$ ( $v$ is the threshold for judging whether the action occurred, $v=2.5$ in our experiment);

3) Start-point positioning. In the obtained MED waveform $(M A)$, we construct an auxiliary positioning waveform to accurately locate the start-point of the motion. We set two points in front of the $P A P$ as turning point $(T P)$ and wave endpoint $W E P$, respectively. The distance from $T P$ to $W E P$ is 20 packages, and the constructed waveform is shown in Fig.6. We calculate the Euclidean distance (ED) between the constructed waveform and the MED waveform and record it, then move the TP forward by 10 packages, and repeat the above steps until 50 iterations are performed. Finally, set the $T P$ at the minimum ED as the start-point of the motion;

4) End-point positioning. Set the start-point we positioned as the beginning of the CSI waveform, initialize the parameters as described in the first step, and calculate the MED as described above. Then use the previous method for positioning the end of the motion. Set the end-point as the beginning of the CSI waveform, and turn to step 1 , until the end of the monitoring.

The performance of our motion segmentation algorithm is 


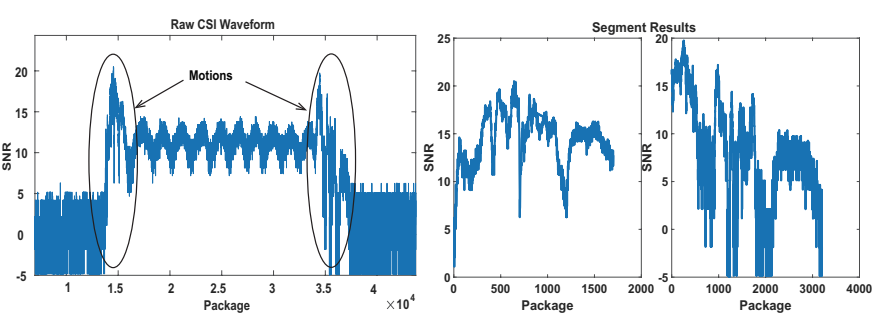

Fig. 7: The performance of our motion segmentation algorithm

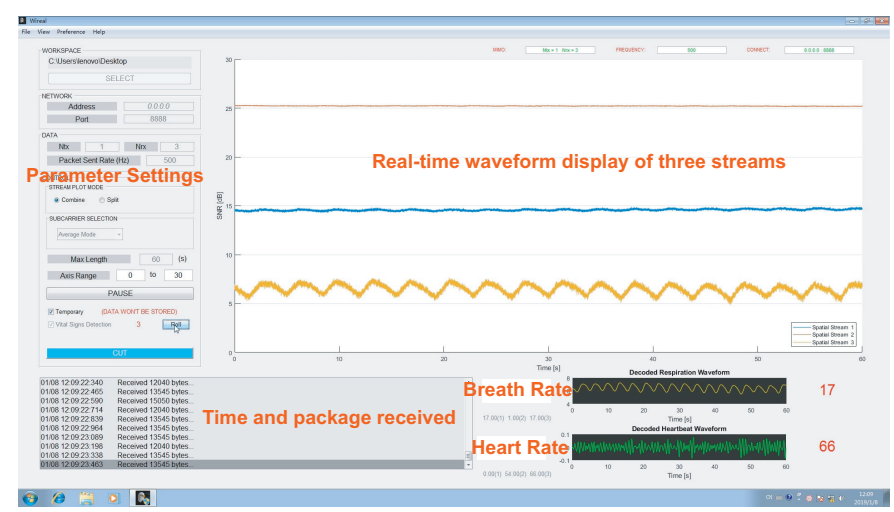

Fig. 8: Real time system's interface.

shown in figure 7, it can segment motionaccurately.

Data Denoising. Received CSI data contains a lot of interference noise due to equipment and environmental factors. In the preprocessing module, we choose the Hampel filter to filter out the outliers which have significantly different values from other neighboring CSI measurements. The goal of the Hampel filter is to identify and replace outliers in a given series. Specifically, we calculate the median of the set consisting of the current CSI sample and its surrounding six samples (three on each side), and use the median absolute deviation to calculate the standard deviation of the set. If the difference between the sample and the median exceeds three times the standard deviation, replace it with the median.

\section{Vital Signs Extracting}

Frequency Domain Segmentation. The trunk deformation caused by the heartbeat is very small, and the CSI change caused by it can be overwhelmed by the change caused by breathing [12]. Therefore, we need to segment them in the frequency domain first, and in this paper, we segment the CSI based on Butterworth bandpass filters and some prior knowledge in the frequency domain (the frequency range related to the normal heartbeat is $60 \mathrm{bpm}$ to $120 \mathrm{bpm}$ which corresponds to $1 \mathrm{~Hz}$ to $2 \mathrm{~Hz}$, the frequency range related to normal breathing is $15 \mathrm{bpm}$ to $30 \mathrm{bpm}$ which corresponds to $0.25 \mathrm{~Hz}$ to $0.5 \mathrm{~Hz}$ ).

Vital Signs Extracting. After segment the CSI in the frequency domain, we extract the heart and respiratory rate by Fast Fourier Transform (FFT). We also design a real-time system to process and display vital signs in real-time use Matlab as shown in Figure. 8.

\section{Performance Evaluation}

In this section, we first verify the effectiveness of our NLOS sensing model, then evaluate the proposed real-time vital signs monitoring system-Wital.

\section{A. Evaluation of The NLOS Sensing Model}

To verify the proposed NLSO sensing model in section .III, we first calculated the Ricean-K value of each stream in setting 1 (the setting as shown in Figure.2a) as shown in Figure. 9, it can be found that the larger the $\mathrm{K}$ is, the worse the sensing capability is. Then we placed a lead sheet between the T1-R1 antenna pair in setting 1 (decrease $\mathrm{K}$ ) to perform a breathing monitoring experiment, the result as shown in Fig.11. It can be found that the motion sensing capability of T1-R1 has been significantly improved. We show the average breathing detection error (BDE), the variance of the CSI waveform (VAR), and the mean amplitude difference (MAD) as Figure.10. We can observe that both the detection accuracy and the sensitivity to motion have improved.

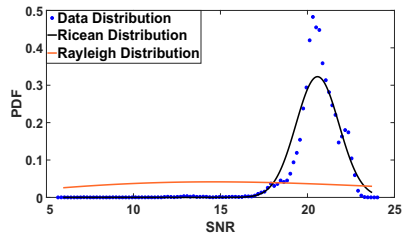

(a)

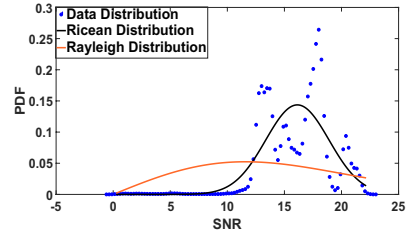

(b)

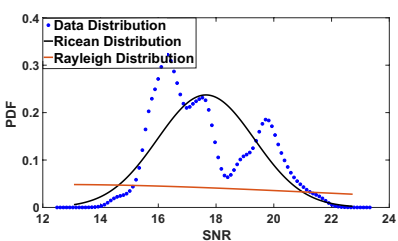

(c)

Fig. 9: Distribution of the three streams in Figure.3a(a)T1$\mathrm{R} 1, \mathrm{~K}=201.1$; (b)T1-R2,K=17.8; (c)T1-R3,K=52.

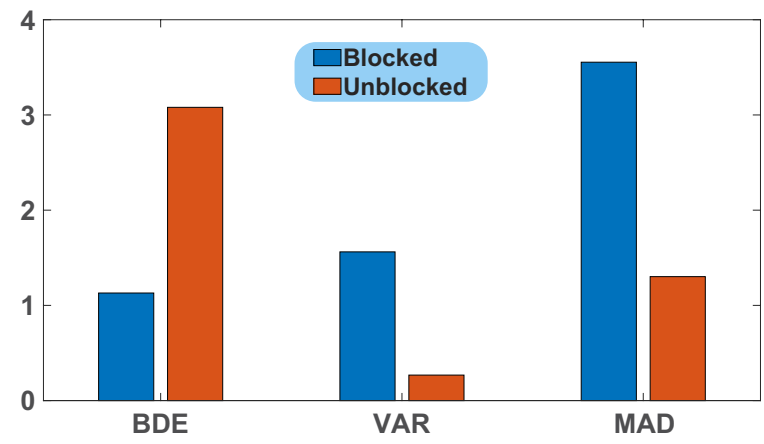

Fig. 10: The average breathing detection error (BDE), the variance of the CSI waveform (VAR), and the mean amplitude difference (MAD) when T1-R1 blocked and unblocked. 


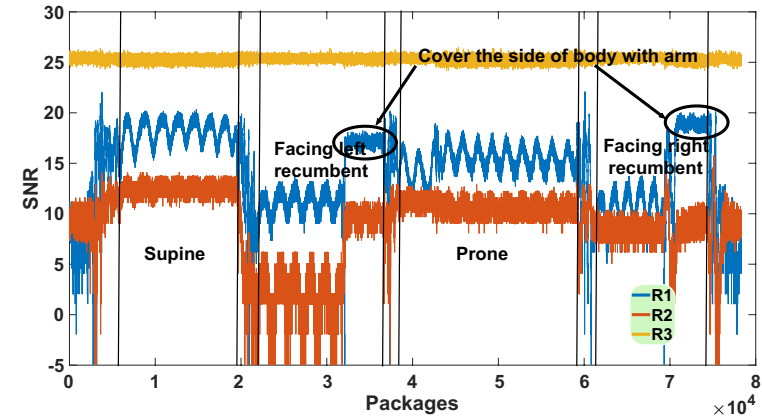

Fig. 11: Obstacle the direct signal from T1 to R1 in setting1, the $\mathrm{K}$ factor of the T1-R1 stream decrease to 12.4 , the $\mathrm{K}$ factor of the T1-R3 stream increase to 5076 .

\section{B. Experimental Setup}

In the actual setup, we build our prototype system based on setting 1 and we choose the T1-R3 stream to monitor vital signs. Due to the limitations of the bed, the person's torso is closer to R3 when lying down or face down; in other words, the torso is far away from the mid-perpendicular of T1-R3. When we are lying down/lying with face down, the chest displacement caused by breath/heartbeat in anteroposterior dimensions still has a significant effect on T1-R3 based on the Fresnel Zone model, it ensures the monitoring performance in different sleeping postures. If we choose and block T1-R1, as shown in Figure.11, when the person is facing left/right recumbent, T1-R1 is sensitive to the breath of the mediolateral dimensions. However, when the person's arm blocks the flank, the monitoring effect becomes poor. It is due to when facing left/right recumbent, the main factor that affects the CSI received by $\mathrm{R} 1$ is the torso deformation of the flank. This is why we chose T1-R3 to monitor vital signs instead of T1-R1.

In the experiments, we place a lead sheet under $\mathrm{T} 1$ to block the LOS path of T1-R3 based on our NLOS sensing mode. CSI is collected use csitool [43], and the receiver transmits the received CSI data to the monitor computer through the network for real-time processing.

We use off-the-shelf hardware devices to implement the proposed system. Specifically, we use two mini PCs as the sending and the receiving devices, and their network cards are Intel Link $5300 \mathrm{WiFi}$ NIC. The miniPCs have a $2.16 \mathrm{GHz}$ Intel Celeron N2830 processor with 2GB RAM and Ubuntu OS in version 12.04. The real-time monitoring computer is a desktop computer equipped with an Intel Core i5 $3450 \mathrm{CPU}$ (3.1G HZ), 2GB storage.

Our real environment settings are shown in Fig. 12, the distance from T1-R3 is $80 \mathrm{~cm}$, we place a lead sheet under T1 to enhance the performance of R3, and we only use the stream T1-R3 to monitor vital signs in the experiments. The purpose of setting the other two antennas is to prepare for future sleep monitoring system expansion (sleep motion recognition such as turning over, sit up; perceive motion direction, and disease alert such as epilepsy).

We experimented in a lab environment as shown in Fig. 12 , a total of ten volunteers participated in the experiments $(6$ males and 4 females) whose age ranges is 21 to 26 . These ten

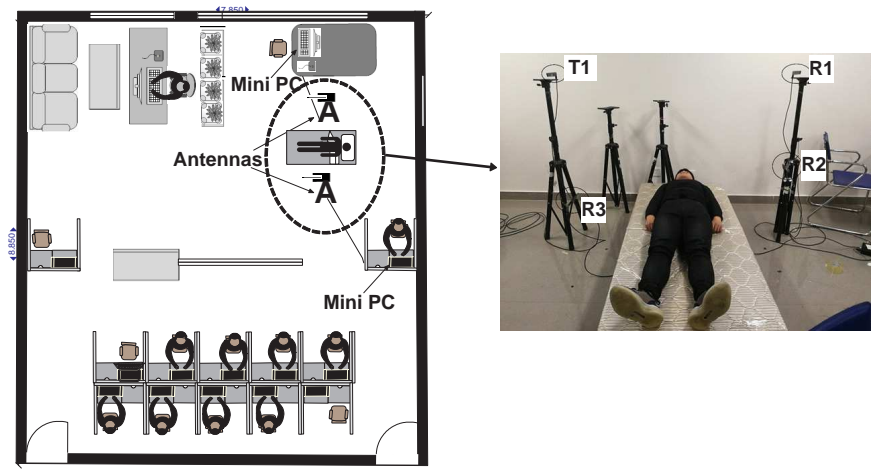

Fig. 12: Prototype System

volunteers were general university students who volunteered for the experiments. In the experiments, we did not limit the normal activities of others in the lab.

Each participant underwent an actual test of 30 minutes in different sleeping postures naturally (prone, supine, facing left recumbent, and facing right recumbent). Different from previous work [12], [17], we did not use a metronome to control the volunteer's respiratory rate, and we do not need to use a directional antenna to monitor heart rate under LOS conditions. The ground truths of breathing and heartbeat are measured by an accelerometer attached to the abdomen and a fingertip pulse oximeter, respectively.

\section{Evaluation Results}

The monitored CSI, which mainly contains the respiratory information obtained by the bandpass filter, is compared with the acceleration sensor data attached to the abdomen, as shown in Fig. 13. We can observe that the CSI waveform is highly consistent with the respiratory waveform obtained by the acceleration sensor. Fig. 14 compares the processed CSI waveform which contains heartbeats to an acceleration sensor's reading attached to the chest, we can found that the occurrence of the heartbeat on the accelerometer is also consistent with the detection result of CSI. These indicate that the CSI obtained from WiFi signals can be used to extract fine-grained heartbeat and respiration information.

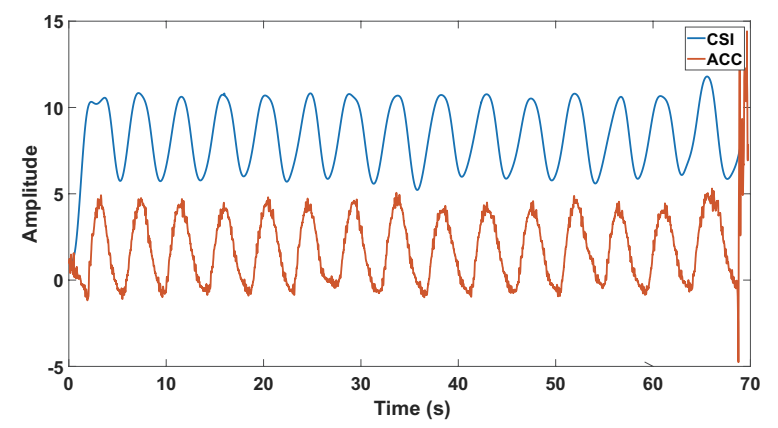

Fig. 13: Comparison of processed CSI and accelerometer (ACC) readings for breathing.

We evaluate the overall performance of breathing and heart rate estimation under different sleep postures, the final result 


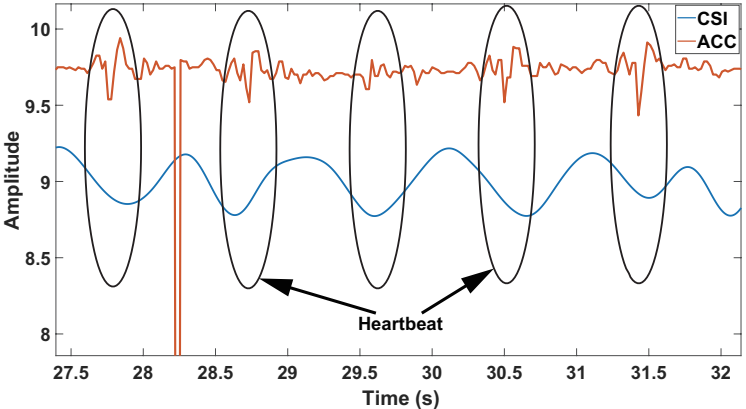

Fig. 14: Comparison of processed CSI and accelerometer (ACC) readings for heartbeat

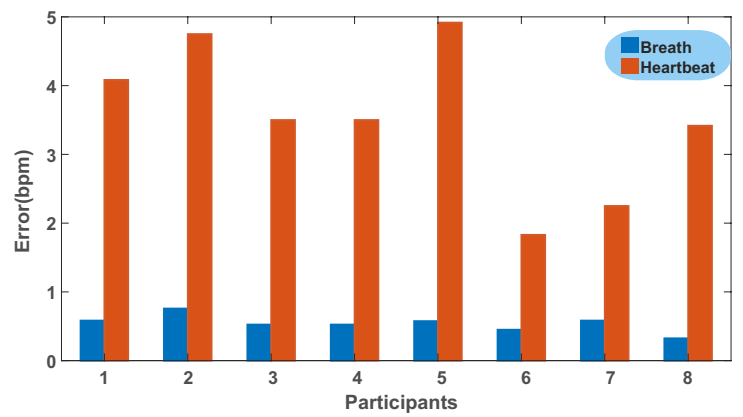

Fig. 15: Performances of different sleep volunteers

is an average error of $0.498 \mathrm{bpm}$ (beat per minute) for detect breathing rate, $3.531 \mathrm{bpm}$ for detect heart rate, and the accuracy is $96.887 \%$ and $94.708 \%$, respectively.

Figure. 15 illustrates the vital signs (breath and heart rate) monitoring error of different participants; volunteers have different body types, which results in different final results. However, in general, our system has high accuracy in detect respiration, and the error in detect heart rate is also within the acceptable range in a non-clinical environment.

Figure. 16 illustrates the vital signs (breath and heart rate) monitoring error of different sleeping postures, in supine and facing right recumbent postures, monitoring error is relatively small. However, for facing left recumbent posture, the error in monitoring heart rate is largest, and for prone posture, the error in monitoring breathing rate is largest. This is because the effective displacement of the dynamic path caused by these two sleeping postures is small, which is not conducive to

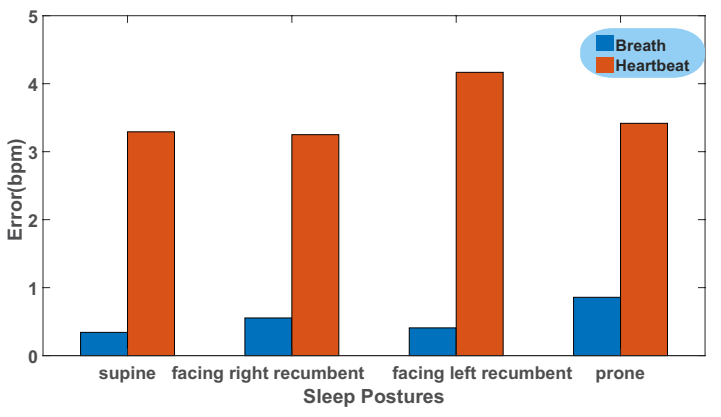

Fig. 16: Performances of different sleep postures vital signs monitoring. In general, our system can accurately monitor vital signs for different sleeping postures.

\section{CONCLUSION}

In this paper, we show that we could use WiFi signals to track breath and heartbeat with different sleeping postures using only one pair of WiFi devices. To achieve this, we propose an NLOS sensing model based on the Ricean-K theory to help monitor the minor displacements caused by breathing and heartbeat, and we theoretically prove that blocking the LOS signal is more beneficial for motion detection in NLOS sensing. We also propose a motion segmentation algorithm based on regularity detection, which can accurately position the range of motions different from vital signs (such as turn over and get up). We implement a real-time prototype system to evaluate our method. The experimental results indicated the performance of our method, and the accuracy is $96.618 \%$ and $94.708 \%$ for breath and heart rate detection, respectively.

\section{REFERENCES}

[1] T. P. Velavan and C. G. Meyer, "The covid-19 epidemic," Tropical medicine \& international health, vol. 25, no. 3, p. 278, 2020.

[2] S. D. Min, J. K. Kim, H. S. Shin, Y. H. Yun, C. K. Lee, and M. Lee, "Noncontact respiration rate measurement system using an ultrasonic proximity sensor," IEEE Sensors Journal, vol. 10, no. 11, pp. 1732$1739,2010$.

[3] P. X. Braun, C. F. Gmachl, and R. A. Dweik, "Bridging the collaborative gap: Realizing the clinical potential of breath analysis for disease diagnosis and monitoring-tutorial," IEEE Sensors Journal, vol. 12, no. 11 , pp. $3258-3270,2012$.

[4] F. L. Facco, D. W. Ouyang, P. C. Zee, and W. A. Grobman, "Sleep disordered breathing in a high-risk cohort prevalence and severity across pregnancy," American journal of perinatology, vol. 31, no. 10, pp. 899904, 2014.

[5] C. A. Kushida, M. R. Littner, T. Morgenthaler, C. A. Alessi, D. Bailey, J. Coleman Jr, L. Friedman, M. Hirshkowitz, S. Kapen, M. Kramer et al., "Practice parameters for the indications for polysomnography and related procedures: an update for 2005," Sleep, vol. 28, no. 4, pp. 499-523, 2005.

[6] T. A. Nappholz, W. N. Hursta, A. K. Dawson, and B. M. Steinhaus, "Implantable ambulatory electrocardiogram monitor," May 19 1992, uS Patent $5,113,869$.

[7] M. Zhao, F. Adib, and D. Katabi, "Emotion recognition using wireless signals," in Proceedings of the 22nd Annual International Conference on Mobile Computing and Networking, 2016, pp. 95-108.

[8] S. Yue, H. He, H. Wang, H. Rahul, and D. Katabi, "Extracting multiperson respiration from entangled rf signals," Proceedings of the ACM on Interactive, Mobile, Wearable and Ubiquitous Technologies, vol. 2, no. 2, pp. 1-22, 2018.

[9] F. Zhang, D. Zhang, J. Xiong, H. Wang, K. Niu, B. Jin, and Y. Wang, "From fresnel diffraction model to fine-grained human respiration sensing with commodity wi-fi devices," Proceedings of the ACM on Interactive, Mobile, Wearable and Ubiquitous Technologies, vol. 2, no. 1, p. 53,2018 .

[10] Y. Zeng, D. Wu, J. Xiong, E. Yi, R. Gao, and D. Zhang, "Farsense: Pushing the range limit of wifi-based respiration sensing with csi ratio of two antennas," Proceedings of the ACM on Interactive, Mobile, Wearable and Ubiquitous Technologies, vol. 3, no. 3, pp. 1-26, 2019.

[11] Y. Gu, Y. Wang, Z. Liu, J. Liu, and J. Li, "Sleepguardian: An rf-based healthcare system guarding your sleep from afar," IEEE Network, pp. $1-8,2020$.

[12] J. Liu, Y. Chen, Y. Wang, X. Chen, J. Cheng, and J. Yang, "Monitoring vital signs and postures during sleep using wifi signals," IEEE Internet of Things Journal, vol. 5, no. 3, pp. 2071-2084, 2018.

[13] H. Wang, D. Zhang, J. Ma, Y. Wang, Y. Wang, D. Wu, T. Gu, and B. Xie, "Human respiration detection with commodity wifi devices: do user location and body orientation matter?" in Proceedings of the 2016 ACM International Joint Conference on Pervasive and Ubiquitous Computing. ACM, 2016, pp. 25-36. 
[14] Y. Zeng, D. Wu, J. Xiong, J. Liu, Z. Liu, and D. Zhang, "Multisense: Enabling multi-person respiration sensing with commodity wifi," Proceedings of the ACM on Interactive, Mobile, Wearable and Ubiquitous Technologies, vol. 4, no. 3, pp. 1-29, 2020.

[15] Y. Gu, J. Zhan, Y. Ji, J. Li, F. Ren, and S. Gao, "Mosense: An rf-based motion detection system via off-the-shelf wifi devices," IEEE Internet of Things Journal, vol. 4, no. 6, pp. 2326-2341, 2017.

[16] X. Liu, J. Cao, S. Tang, J. Wen, and P. Guo, "Contactless respiration monitoring via off-the-shelf wifi devices," IEEE Transactions on Mobile Computing, vol. 15, no. 10, pp. 2466-2479, 2016.

[17] D. Zhang, Y. Hu, Y. Chen, and B. Zeng, "Breathtrack: Tracking indoor human breath status via commodity wifi," IEEE Internet of Things Journal, vol. 6, no. 2, pp. 3899-3911, 2019.

[18] J. Liu, Y. Zeng, T. Gu, L. Wang, and D. Zhang, "Wiphone: Smartphonebased respiration monitoring using ambient reflected wifi signals," Proceedings of the ACM on Interactive, Mobile, Wearable and Ubiquitous Technologies, vol. 5, no. 1, pp. 1-19, 2021.

[19] Y. Ma, G. Zhou, and S. Wang, "Wifi sensing with channel state information: A survey," ACM Computing Surveys (CSUR), vol. 52, no. 3 , pp. 1-36, 2019.

[20] Y. Gu, X. Zhang, Z. Liu, and F. Ren, "Besense: Leveraging wifi channel data and computational intelligence for behavior analysis," IEEE Computational Intelligence Magazine, vol. 14, no. 4, pp. 31-41, 2019.

[21] Y. Gu, Y. Wang, T. Liu, Y. Ji, Z. Liu, P. Li, X. Wang, X. An, and F. Ren, "Emosense: Computational intelligence driven emotion sensing via wireless channel data," IEEE Transactions on Emerging Topics in Computational Intelligence, 2019.

[22] H. Huang and S. Lin, "Widet: Wi-fi based device-free passive person detection with deep convolutional neural networks," Computer Coтmunications, vol. 150, pp. 357-366, 2020.

[23] H. F. T. Ahmed, H. Ahmad, and C. Aravind, "Device free human gesture recognition using wi-fi csi: A survey," Engineering Applications of Artificial Intelligence, vol. 87, p. 103281, 2020.

[24] L. Zhao, H. Huang, X. Li, S. Ding, H. Zhao, and Z. Han, "An accurate and robust approach of device-free localization with convolutional autoencoder," IEEE Internet of Things Journal, vol. 6, no. 3, pp. 58255840, 2019.

[25] M. Abbas, M. Elhamshary, H. Rizk, M. Torki, and M. Youssef, "Wideep: Wifi-based accurate and robust indoor localization system using deep learning," in 2019 IEEE International Conference on Pervasive Computing and Communications (PerCom. IEEE, 2019, pp. 1-10.

[26] Y. Cao, F. Wang, X. Lu, N. Lin, B. Zhang, Z. Liu, and S. Sigg, "Contactless body movement recognition during sleep via wifi signals," IEEE Internet of Things Journal, 2019.

[27] Y. Bai, Z. Wang, K. Zheng, X. Wang, and J. Wang, "Widrive: Adaptive wifi-based recognition of driver activity for real-time and safe takeover," in 2019 IEEE 39th International Conference on Distributed Computing Systems (ICDCS). IEEE, 2019, pp. 901-911.

[28] F. Wang, S. Zhou, S. Panev, J. Han, and D. Huang, "Person-in-wifi: Fine-grained person perception using wifi," in Proceedings of the IEEE International Conference on Computer Vision, 2019, pp. 5452-5461.

[29] D. WU, R. GAO, Y. ZENG, J. LIU, L. WANG, T. GU, and D. ZHANG, "Fingerdraw: Sub-wavelength level finger motion tracking with wifi signals," Proc. ACM Interact. Mob. Wearable Ubiquitous Technol, vol. 1, no. 1, 2020.

[30] Y. Meng, J. Li, H. Zhu, X. Liang, Y. Liu, and N. Ruan, "Revealing your mobile password via wifi signals: Attacks and countermeasures," IEEE Transactions on Mobile Computing, 2019.

[31] H. Aly and M. Youssef, "Zephyr: Ubiquitous accurate multi-sensor fusion-based respiratory rate estimation using smartphones," in IEEE INFOCOM 2016-The 35th Annual IEEE International Conference on Computer Communications. IEEE, 2016, pp. 1-9.

[32] J. Paalasmaa, M. Waris, H. Toivonen, L. Leppäkorpi, and M. Partinen, "Unobtrusive online monitoring of sleep at home," in 2012 Annual International Conference of the IEEE Engineering in Medicine and Biology Society. IEEE, 2012, pp. 3784-3788.

[33] M. Kumar, A. Veeraraghavan, and A. Sabharwal, "Distanceppg: Robust non-contact vital signs monitoring using a camera," Biomedical optics express, vol. 6, no. 5, pp. 1565-1588, 2015.

[34] J. Salmi and A. F. Molisch, "Propagation parameter estimation, modeling and measurements for ultrawideband mimo radar," IEEE Transactions on Antennas and Propagation, vol. 59, no. 11, pp. 4257-4267, 2011.

[35] N. Patwari, L. Brewer, Q. Tate, O. Kaltiokallio, and M. Bocca, "Breathfinding: A wireless network that monitors and locates breathing in a home," IEEE Journal of Selected Topics in Signal Processing, vol. 8, no. 1 , pp. 30-42, 2013.
[36] N. Patwari, J. Wilson, S. Ananthanarayanan, S. K. Kasera, and D. R. Westenskow, "Monitoring breathing via signal strength in wireless networks," IEEE Transactions on Mobile Computing, vol. 13, no. 8, pp. 1774-1786, 2013.

[37] H. Abdelnasser, K. A. Harras, and M. Youssef, "Ubibreathe: A ubiquitous non-invasive wifi-based breathing estimator," in Proceedings of the 16th ACM International Symposium on Mobile Ad Hoc Networking and Computing. ACM, 2015, pp. 277-286.

[38] C. Wu, Z. Yang, Z. Zhou, X. Liu, Y. Liu, and J. Cao, "Non-invasive detection of moving and stationary human with wifi," IEEE Journal on Selected Areas in Communications, vol. 33, no. 11, pp. 2329-2342, 2015.

[39] C. Chen, Y. Han, Y. Chen, H.-Q. Lai, F. Zhang, B. Wang, and K. R. Liu, "Tr-breath: Time-reversal breathing rate estimation and detection," IEEE Transactions on Biomedical Engineering, vol. 65, no. 3, pp. 489-501, 2017.

[40] Y. Gu, X. Zhang, Z. Liu, and F. Ren, "Wifi-based real-time breathing and heart rate monitoring during sleep," in 2019 IEEE Global Communications Conference (GLOBECOM). IEEE, 2019, pp. 1-6.

[41] F. Zhang, K. Niu, J. Xiong, B. Jin, T. Gu, Y. Jiang, and D. Zhang, "Towards a diffraction-based sensing approach on human activity recognition," Proceedings of the ACM on Interactive, Mobile, Wearable and Ubiquitous Technologies, vol. 3, no. 1, pp. 1-25, 2019.

[42] C. Tepedelenlioglu, A. Abdi, and G. B. Giannakis, "The ricean k factor: estimation and performance analysis," IEEE Transactions on Wireless Communications, vol. 2, no. 4, pp. 799-810, 2003.

[43] D. Halperin, W. Hu, A. Sheth, and D. Wetherall, "Tool release: Gathering 802.11n traces with channel state information," ACM SIGCOMM CCR, vol. 41, no. 1, p. 53, Jan. 2011. 